\title{
Sulfur Annulated Hexa-peri-hexabenzocoronene Decorated with Phenylthio Groups at the Periphery
}

\author{
Yuan-Zhi Tan, ${ }^{[a, c]}$ Silvio Osella, ${ }^{[b]}$ Yi Liu, ${ }^{[a]}$ Bo Yang,${ }^{[a]}$ David Beljonne ${ }^{[b]}$ Xinliang Feng, ${ }^{\left[{ }^{[a, d]}\right.}$ and Klaus \\ Müllen ${ }^{\star[a]}$
}

\begin{abstract}
The chemical nature of edge periphery essentially determines the physical properties of graphene. As a molecular-level model system, large polycyclic aromatic hydrocarbons, i.e. so-called nanographenes, can be chemically modified through either edge functionalization or doping with heteroatoms. Although the synthetic protocols for edge substitution are well developed, incorporation with heteroatoms by the bay annulation of large PAHs remains an enormous challenge. In this study, we present a feasible peripheral sulfur annulation of hexa-peri-hexabenzocoronene (HBC) by thiolation of perchlorinated HBC. The tri-sulfur-annulated HBC and di-sulfur-annulated HBC decorated with phenylthio groups were obtained and characterized by X-ray diffraction, revealing their distinct sulfur-annulated peripheral structure. Associated with theoretical calculations, we propose that the regioselective sulfur annulation results from the minimization of strain in the aromatic backbone. We further demonstrate the structure-correlated property modulation by sulfur annulation, manifesting decrease in band gap and tunable redox activity.
\end{abstract}

The peripheral structure of graphene plays a key role in governing its fundamental physical properties. The chemical functionalization of graphene, either at its periphery or on the inplane surface, provides a feasible means to modulate its chemical nature, as well as its associated electronic properties. ${ }^{[1]}$ Polycyclic aromatic hydrocarbons (PAHs) are regarded as segments of infinite two-dimensional graphene (especially the large PAHs, which are also generally named nanographenes). ${ }^{[2]}$ Thus, PAHs can serve as an ideal molecular-level model for understanding and predicting the chemical reactivity of graphene. For instance, Friedel-Crafts reaction, nucleophilic chlorination, which was originally developed for PAHs, can also be adopted for the chemical functionalization of graphene. ${ }^{[3]}$ In the past decade, various functional PAHs, such as hexa-perihexabenzocoronenes (HBCs) with tailor-made p- or n-type semiconducting properties, have been achieved by varying the electron-donating or -withdrawing substitutions. The introduction

[a] Dr. Y. Z. Tan, Y.Liu, B. Yang, Prof. Dr. X.L. Feng, Prof. Dr. K. Müllen Max Planck Institute for Polymer Research, Ackermannweg 10, D55128, Mainz, Germany

E-mail: muellen@mpip-mainz.mpg.de

[b] Dr. S. Osella, Prof. Dr. D. Beljonne

Laboratory for Chemistry of Novel Materials, University of Mons, Place du Parc 20, B-7000 Mons, Belgium

[c] Dr. Y.Z. Tan

State Key Laboratory for Physical Chemistry of Solid Surfaces and Department of Chemistry, College of Chemistry and Chemical Engineering, Xiamen University, 361005, Xiamen, China

[d] Prof. Dr. X. L. Feng

Center for Advancing Electronics Dresden (cfaed) \& Department of Chemistry and Food Chemistry, Technische Universität Dresden, 01062 Dresden, Germany

Email: xinliang.feng@tu-dresden.de

Supporting information for this article is given via a link at the end of the document. of alkyl chains with different amphiphilicities at the periphery of HBCs has led to highly stable discotic liquid crystals ${ }^{[4]}$ and unique supramolecular nanostructures (such as nanofibers and nanotubes) ${ }^{[5]}$ In contrast, the bay annulation of PAHs with heteroatoms, such as sulfur ${ }^{[6]}$ and nitrogen, ${ }^{[7]}$ at the periphery of PAHs might impact not only on their molecular planarity and conformation but also their chemical reactivity and electronic structures, ${ }^{[8]}$ such as stronger dipolar interactions, an enhanced electron-donating ability, and less aromaticity. Several sulfurannulated PAHs have been reported to exhibit unique redox behavior and superior charge transporting properties. ${ }^{[6,8]}$ Nevertheless, their syntheses are so far only limited to a few cases of small PAHs, such as perylene ${ }^{[6 \mathrm{~b}, 6 \mathrm{~d}]}$ and triphenylene. ${ }^{[6 a, 6 \mathrm{e}]}$ For the large PAHs, which are more related to graphene, the sulfur annulation at the periphery remains a challenge, most likely due to the lack of suitable synthetic protocols.

a

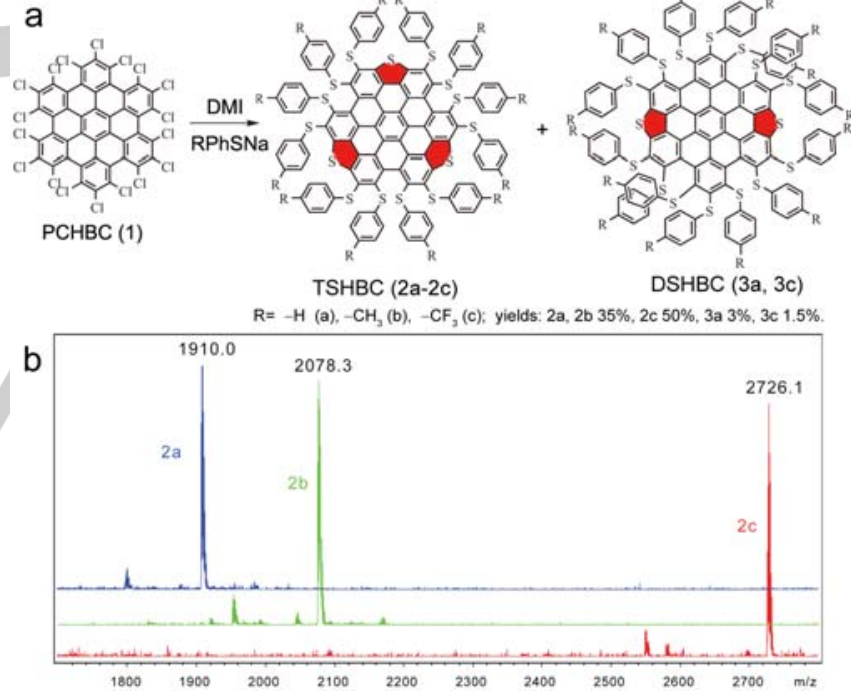

Figure 1. Synthesis of TSHBC (2a-2c) and DSHBC (3a, 3c). a. Thiolation reaction scheme for PCHBC. The thiophene rings in TSHBC and DSHBC were highlighted in red. b. Mass spectra of TSHBC with different peripheral phenylthio groups. The mass spectra of $\mathbf{2 a}, \mathbf{2} \mathbf{b}$ and $\mathbf{2 c}$ are plotted in blue, green and red, respectively. DMI = 1,3-dimethyl-2-imidazolidinone.

Here, we demonstrate the unprecedented tri-sulfurannulated HBC (TSHBC, 2a-2c) with twelve phenylthio groups decorated at the periphery (Figure 1a), achieved by thiolation of perchlorinated HBC (PCHBC, 1). The structure of TSHBC was unambiguously determined using single crystal $\mathrm{X}$-ray diffraction, showing three annulated sulfur atoms symmetrically at bay positions of parent HBC in an alternating manner (Figure 1a). We were further able to capture the di-sulfur-annulated HBC (DSHBC, 3a, 3c) (Figure 1a), which has two annulated sulfurs at opposite bay regions and fourteen phenylthio groups, as a by- 
product. In association with structural analyses and theoretical calculations, we propose that the minimization of strain in the aromatic skeleton accounts for the regioselective sulfur annulation. Compared with pristine HBC, TSHBC shows a remarkable red shift in its UV-vis absorption and photoluminescence by 100 and $80 \mathrm{~nm}$, respectively. Moreover, TSHBC exhibits three reversible reduction processes, which can be modulated by the electron-withdrawing or -donating property of phenylthio substituents.

Figure 1a illustrates the synthetic route towards TSHBC (2a2c) by thiolation of PCHBC (1) (Figure 1a, see Supporting Information S1 for synthesis details). Notably, in contrast to the reported thiolation of perchlorinated aromatic compounds, ${ }^{[9-11]}$ not only nucleophilic substitution of peripheral chlorines by phenyl thiolate occurs but also symmetrical annulation of three sulfur atoms occurs by forming three nonadjacent thiophene rings at the bay-regions of $\mathrm{HBC}$ (Figure 1). Moreover, we found that this reaction was tolerant to different phenyl thiolates with electron-donating or -withdrawing natures, such as phenyl thiolate, 4-methylphenyl thiolate, and 4-trifluoromethylphenyl thiolate, producing dodecakis(phenylthio)-TSHBC (2a), dodecakis(4-methylphenylthio)-TSHBC (2b) and dodecakis(4trifluoromethylphenylthio)-TSHBC (2c) with similar isolated yields (Figure 1a). Considering that eighteen sulfur-carbon bonds are formed in one reaction, the achieved yield is remarkable ( $~ 95 \%$ yield for each C-S bond formation).

As shown in Figure 1b, the mass spectra display one dominant peak, which perfectly matches the structural formula of the corresponding TSHBC. The small peaks identifiable in the mass spectra of $\mathbf{2 a - 2 c}$ are assigned to the fragmentation, in which it loses one phenylthio group during the ionization process in the measurement. The ${ }^{1} \mathrm{H}$ NMR spectrum of $\mathbf{2 a}$ shows two sets of proton signals with chemical shifts at 7.5-7.1 ppm and 6.7-6.1 ppm, corresponding to the phenylthio groups at the vertex and at the bay positions of $\mathbf{2 a}$, respectively (Figures S1S2). The ${ }^{1} \mathrm{H}$ NMR spectra of $\mathbf{2} \mathbf{b}$ and $\mathbf{2} \mathbf{c}$ also exhibit two sets of proton signals resulting from the peripheral substituted phenylthio groups, confirming their identical chemical structures with $\mathbf{2 a}$ (Figures S3-S6).

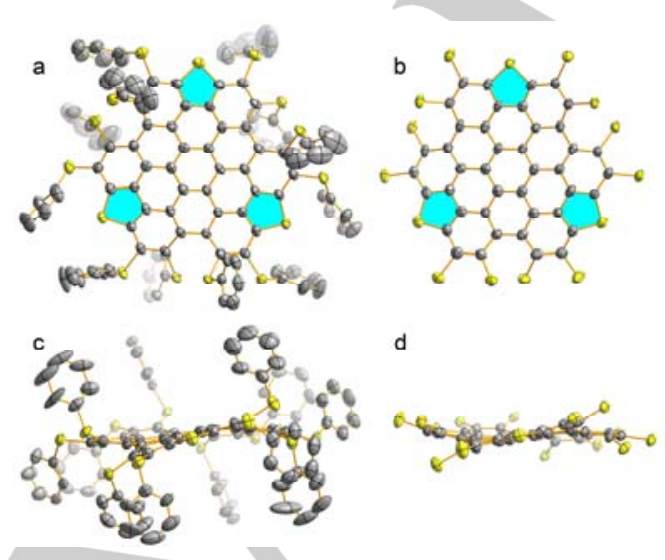

Figure 2. Crystal structure of $\mathbf{2 a}$. Top view with and without phenyl groups (a. b). Side view with and without phenyl groups (c. d). Thiophene rings are highlighted in cyan.
Exemplified by $\mathbf{2 a}$, the unambiguous structure of TSHBC was assessed by single crystal X-ray diffraction (see Supporting Information S3). As depicted in Figure 2, the most distinguishable feature of $\mathbf{2} \mathbf{a}$ is three symmetrically annulated sulfurs at the bay positions of parent HBC. $2 a$ has a quasi $C_{3 v}$ symmetry without considering the conformation of the peripheral phenylthio groups (Figure 2b) and exhibits a curved $\pi$ conjugated framework, resulting from the congestion of its steric bulky phenylthio groups. The average torsion angle at the three bay positions in $\mathbf{2 a}$ is $26.5^{\circ}$, comparable with that of perthiolated coronene $\left(23.3^{\circ}\right) .^{[12]}$ However, the inner core of $\mathbf{2 a}$ is obviously more planar than the PCHBC (its torsion angle at the bay position is $\left.53^{\circ}\right) .{ }^{[3 c]}$ This result can be attributed to the formation of three thiophene rings at the bay positions without involving sterically congested peripheral groups (Figure 2).

Apart from the annulation of sulfur at the bay positions, twelve phenylthio groups are decorated at the periphery of $\mathbf{2 a}$. The conformation of the phenylthio groups adopts an up and down manner to minimize steric hindrance. There is no $\pi-\pi$ stacking between the inner aromatic core of $\mathbf{2 a}$ in the crystal packing, similar to permethoxylated $\mathrm{HBC}^{[13]}$ and $\mathrm{PCHBC} .{ }^{[3 c]}$ Interestingly, together with the inner aromatic plane, the phenylthio groups at the edge form a hydrophobic pocket, which can serve as a supramolecular host for other components. As revealed in the crystal packing of $\mathbf{2 a}$, this hydrophobic pocket can host up to three phenyl groups from neighboring molecules, assembling $\mathbf{2 a}$ into a columnar superstructure in the solid state (Figure S7).
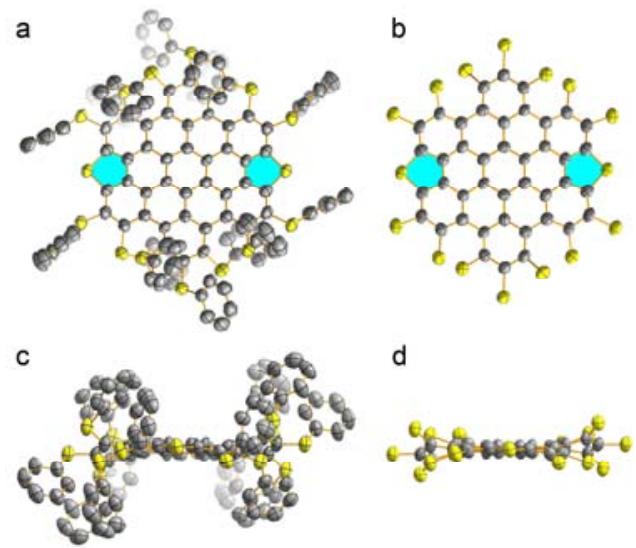

Figure 3. Crystal structure of 3a. Top view with and without phenyl groups (a, b). Side view with and without phenyl groups (c, d). Thiophene rings are highlighted in cyan.

For the mechanism of sulfur annulation at the bay position, we propose that when a phenyl thiolate group replaces one of chlorines at the bay position, it can sequentially attack the neighboring chlorine to form a ring-closure intermediate, in which the bridging sulfur becomes positive nature; then a chlorobenzene is released to recover the aromaticity and the neutral sulfur, resulting in sulfur annulation at the bay position (see Supporting Information S4). Similar sulfur annulation was also reported in a Stille type reaction of tetrachloroperylene 
bisimide. ${ }^{[14]}$ However, of interest is the regioselectivity of sulfur annulation in TSHBC. We succeeded in the isolation of the disulfur annulated HBC (DSHBC) (Figure 1a) from the reaction products as the byproduct (Figure S8). The structure of $\mathbf{3 a}$ was unambiguously characterized by single crystal X-ray diffraction (see Supporting Information S3). As presented in Figure 3, the structure of $\mathbf{3 a}$ clearly shows two annulated sulfurs at the opposite bay regions, forming two thiophene rings in the aromatic skeleton. $\mathbf{3 a}$ is further substituted with fourteen phenylthio groups at the periphery, which were introduced by the nucleophilic substitution of chlorines. The phenylthio groups in 3a also adopt an up and down conformation to reduce the congestion (Figure 3).

One can rule out the possibility that DSHBC is the intermediate for the formation of TSHBC. Nevertheless, the common structural feature between TSHBC and DSHBC (Figures 2 and 3 ) is that further sulfur annulation at the remainder bay positions leads to the formation of adjacent thiophene rings. Adjacent thiophene rings can make the conjugated skeleton curved as in the case of bowl-shaped triphenylenotrithiophene, ${ }^{[6 a, 6 \mathrm{e}]}$ i.e., tri-sulfur annulated triphenylene, which has three adjacent thiophene rings at the periphery. Due to the high strain on the curved aromatic core ${ }^{[15]}$ sulfur annulation at the adjacent bay position is energetically unfavorable, which is confirmed by theoretical calculations (vide infra). Thus, we conceive that the sulfur annulation prefers to occur at nonadjacent bay positions, which is the case for both TSHBC and DSHBC. As with the TSHBC, the DSHBC also exhibits a decreased torsion angle (25.8 $)$ compared with fully substituted HBCs. ${ }^{[3 c, 13]}$ This result suggests that the formation of thiophene rings in the TSHBC and the DSHBC releases the unfavorable strain resulting from the steric hindrance of congest phenylthio groups, which is also confirmed by density functional theory (DFT) calculations.

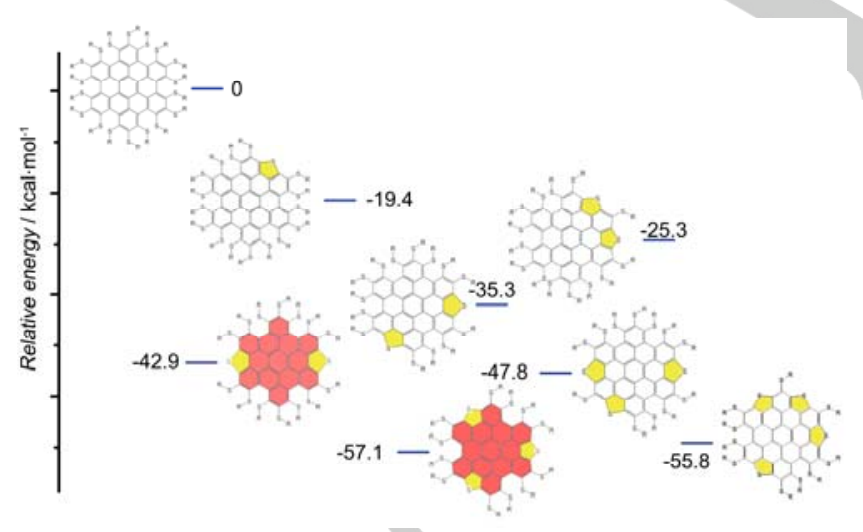

Figure 4. Relative stability of possible products as obtained from DFT. The thiophene rings are highlighted in yellow. The isolated products from the reaction are marked in red. $\mathrm{R}$ is equal to a phenyl group.

To support our proposed mechanism of regioselectivity, we performed DFT calculations with the HSE functional ${ }^{[16]}$ and 6$31 \mathrm{G}^{*}$ basis sets ${ }^{[17]}$ (see Supporting Information S6). $\cdot$ A series of possible products with different numbers of annulated sulfur were fully optimized, and their relative stability was evaluated (Figure 4). We found that perthiolated HBC without any annulated sulfur at the bay position had the highest energy among all of the possible products $(57.1 \mathrm{kcal} / \mathrm{mol}$ higher than the most stable one, i.e., the major product $2 a$ ) (Figure 4). This result strongly suggests that the sulfur annulation at the bay regions releases the strain on the carbon framework. In addition, as revealed in Figure 4, the sulfur annulation at adjacent bay positions is also energetically less favorable compared with other annulation patterns. For instance, among three isomers of di-sulfur-annulated $\mathrm{HBC}$, the isomer with two sulfurs annulated at adjacent bay positions is $17.6 \mathrm{kcal} / \mathrm{mol}$ less stable than the captured DSHBC (Figure 4). Moreover, further sulfur annulation of the DSHBC only results in a modest decrease in energy, by only $5.1 \mathrm{kcal} / \mathrm{mol}$. In contrast, further sulfur annulation at the nonadjacent bay position in the corresponding di-sulfurannulated intermediate of TSHBC is more favorable in energy by $21.8 \mathrm{kcal} / \mathrm{mol}$ and further sulfur annulation of TSHBC is also not energetically favourable (Figure 4). Altogether, one can conclude that the minimization of skeleton strain by nonadjacent sulfur annulation accounts for the formation of TSHBC.
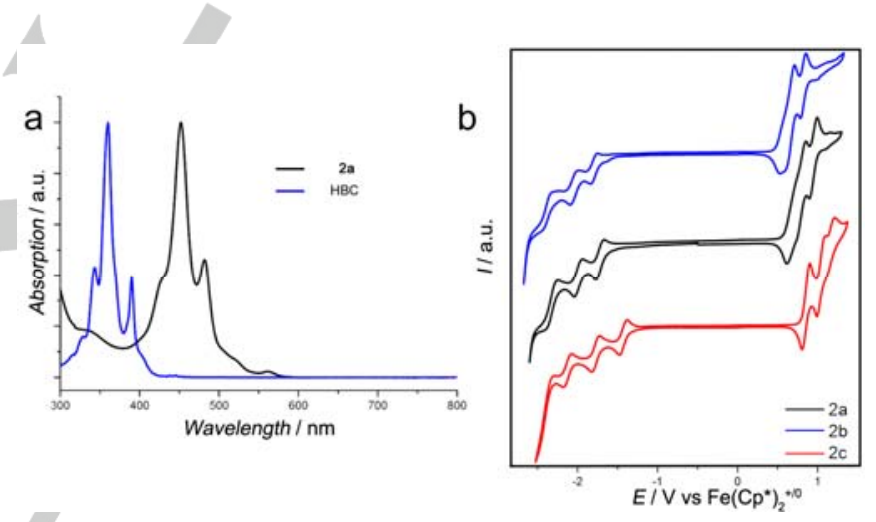

Figure 5. Optical and electrochemical properties of TSHBC. a. UV-Vis spectra of HBC (blue) and $2 a$ (black). b. Cyclic voltammograms of $2 a$ (black), $2 b$ (blue) and $2 \mathrm{c}$ (red).

Compounds $\mathbf{2 a - 2 c}$ are highly soluble in common organic solvents such as toluene, chloroform and dichloromethane (> 50 $\mathrm{mg} / \mathrm{ml}$ in toluene). The UV-vis spectrum of $\mathbf{2 a}$ displays three absorption bands that peak at 452, 482 and $560 \mathrm{~nm}$, which can be assigned to the $\beta, p$ and $\alpha$ bands, respectively (Figure 5). An optical band gap of $2.57 \mathrm{eV}$ can be calculated for $2 \mathrm{a}$. Compared with that of unsubstituted $\mathrm{HBC}{ }^{[18]} \mathbf{2 a}$ exhibits nearly the same absorption profile, but it is red shifted by $100 \mathrm{~nm}$, indicating a decreased optical bandgap by $0.5 \mathrm{eV}$, attributable to the dense edge functionalization and extended $\pi$ system of $\mathbf{2 a}$. Timedependent DFT calculations indicate strong configurational mixing associated with near degeneracy of the frontier molecular orbitals (Figures S9-S12, Tables S2-S5 and see Supporting Information S6 for calculation details) and a bathochromic shift of $\sim 110 \mathrm{~nm}$ transitioning from HBC to TSHBC, in good agreement with the experimental data. The photoluminescence of $2 \mathbf{a}$ shows a concentration-independent emission with $\lambda_{\max }$ located at $568 \mathrm{~nm}$, approximately $80 \mathrm{~nm}$ red-shifted compared with that of the unsubstituted $\mathrm{HBC} .^{[18]}$ The absorption and 
photoluminescence spectra of $\mathbf{2} \mathbf{b}$ and $\mathbf{2} \mathbf{c}$ are almost identical to that of $\mathbf{2} \mathbf{a}$, which indicates that the electron-withdrawing or donating phenylthio group has a negligible effect on the optical properties of TSHBC (Figures S13, S14).

Sulfur-containing organic compounds have been extensively studied as components of conducting organic materials, ${ }^{[19]}$ e.g., charge transfer salts. All of the compounds (2a-2c) provided three reversible one-electron reduction waves with similar profiles (Figure $5 b$ and Table S6). Remarkably, the first reduction potential for $2 \mathrm{a}\left(-1.72 \mathrm{~V}\right.$ vs $\left.\mathrm{FeCp}_{2}{ }^{+/ 0}\right)$ is $0.94 \mathrm{~V}$ more positive than that for an alkyl chain substituted $\mathrm{HBC}^{[20]}$ and 0.24 $\mathrm{V}$ more positive than that for a dodeca-fluorine substituted HBC. ${ }^{[21]}$ This result can be explained by the capability of annulated sulfurs and peripheral phenylthio groups to stabilize the negative charges of the reduced species. Interestingly, the electrochemical property of TSHBC is quite sensitive to the substituted phenylthio groups. The first reduction potential of $\mathbf{2 c}$ exhibits a positive shift of $0.30 \mathrm{~V}$ in comparison with that of $\mathbf{2 a}$, given that the strong electron-withdrawing trifluoromethyl groups can further stabilize the anion state of TSHBC. In contrast, $\mathbf{2} \mathbf{b}$ possesses a weak electron-donating methyl effect; thus, is shows a more negative reduction potential by $0.07 \mathrm{~V}$ compared with those of $\mathbf{2 a}$. In the oxidation direction, both $\mathbf{2 a}$ and $\mathbf{2} \mathbf{b}$ manifest irreversible and multi-electron oxidation processes, whereas $\mathbf{2 c}$ shows two reversible oxidation processes.

In summary, we have presented the first case of sulfurannulated $\mathrm{HBC}$ at the bay region by thiolation of $\mathrm{PCHBC}$. The strain minimization of the aromatic carbon skeleton should be responsible for the regioselective sulfur annulation at the bay positions during thiolation. Notably, the sulfur annulation can serve as an efficient route to modulate the optical and electrochemical properties of PAHs. This synthetic protocol provides a new scaffold for constructing discotic functional materials and also opens the possibility of peripheral sulfurannulated graphene materials in terms of thiolation reactions on edge-chlorinated graphenes and graphene nanoribbons. The tunable electrochemical properties achieved by tailoring the peripheral thiol groups will further facilitate the design of redox sensors and organic conductors based on peripheral sulfurated graphene materials.

\section{Acknowledgements}

This work was financially supported by an ERC grant on NANOGRAPH, DFG Priority Program SPP 1459, EU Project GENIUS, MoQuas, UPGRADE and Graphene Flagship. DB is Research Director of FNRS Belgium.

Keywords: Sulfur, Annulation, Functionalization, HBC, Thiolation

[1] a) L. Yan, Y. B. Zheng, F. Zhao, S. Li, X. Gao, B. Xu, P. S. Weiss, Y. Zhao, Chem. Soc. Rev. 2012, 41, 97-114; b) V. Georgakilas, M. Otyepka, A. B. Bourlinos, V. Chandra, N. Kim, K. C. Kemp, P. Hobza, R. Zboril, K. S. Kim, Chem. Rev. 2012, 112, 6156-6214; c) E. Bekyarova,
S. Sarkar, F. Wang, M. E. Itkis, I. Kalinina, X. Tian, R. C. Haddon, Acc. Chem. Res. 2013, 46, 65-76

[2] J. Wu, W. Pisula, K. Müllen, Chem. Rev. 2007, 107, 718-747.

[3] a) S.-Y. Bae, I.-Y. Jeon, J. Yang, N. Park, H. S. Shin, S. Park, R. S. Ruoff, L. Dai, J.-B. Baek, ACS Nano 2011, 5, 4974-4980; b) I.-Y. Jeon, D.-S. Yu, S.-Y. Bae, H.-J. Choi, D.-W. Chang, L.-M. Dai, J.-B. Baek, Chem. Mater. 2011, 23, 3987-3992; c) Y.-Z. Tan, B. Yang, K. Parvez, A. Narita, S. Osella, D. Beljonne, X. Feng, K. Müllen, Nat. Commun. 2013 4, 3646/3641-3646/3647.

[4] a) S. Sergeyev, W. Pisula, Y. H. Geerts, Chem. Soc. Rev. 2007, 36, 1902-1929; b) S. Kumar, Chem. Soc. Rev. 2006, 35, 83-109.

[5] a) S. Laschat, A. Baro, N. Steinke, F. Giesselmann, C. Haegele, G. Scalia, R. Judele, E. Kapatsina, S. Sauer, A. Schreivogel, M. Tosoni, Angew. Chem., Int. Ed. 2007, 46, 4832-4887; Angew. Chem. 2007, 119, 4916-4973; b) X. Feng, V. Marcon, W. Pisula, M. R. Hansen, J. Kirkpatrick, F. Grozema, D. Andrienko, K. Kremer, K. Müllen, Nat. Mater. 2009, 8, 421-426; c) J. P. Hill, W. Jin, A. Kosaka, T. Fukushima, H. Ichihara, T. Shimomura, K. Ito, T. Hashizume, N. Ishii, T. Aida, Science 2004, 304, 1481-1483; d) W. Jin, Y. Yamamoto, T. Fukushima, N. Ishii, J. Kim, K. Kato, M. Takata, T. Aida, J. Am. Chem. Soc. 2008, 130, $9434-9440$

[6] a) K. Imamura, K. Takimiya, T. Otsubo, Y. Aso, Chem. Commun. 1999 1859-1860; b) J. Lawson, R. DuVernet, V. Boekelheide, J. Amer. Chem. Soc. 1973, 95, 956-957; c) W. Jiang, Y. Zhou, H. Geng, S.-D. Jiang, S.K. Yan, W.-P. Hu, Z.-H. Wang, Z.-G. Shuai, J. Pei, J. Am. Chem. Soc. 2011, 133, 1-3; d) Y. Sun, L. Tan, S. Jiang, H. Qian, Z. Wang, D. Yan, C. Di, Y. Wang, W. Wu, G. Yu, S. Yan, C. Wang, W. Hu, Y. Liu, D. Zhu, J. Am. Chem. Soc. 2007, 129, 1882-1883; e) X. Li, Y. Zhu, J. Shao, B. Wang, S. Zhang, Y. Shao, X. Jin, X. Yao, R. Fang, X. Shao, Angew. Chem., Int. Ed. 2014, 53, 535-538; Angew. Chem. 2014, 126, 545 548.

[7] a) Q. Tan, S. Higashibayashi, S. Karanjit, H. Sakurai, Nat. Commun 2012, 3, 1891-1895; b) J. Wei, B. Han, Q. Guo, X. Shi, W. Wang, N. Wei, Angew. Chem., Int. Ed. 2010, 49, 8209-8213; Angew. Chem. 2010, 49, 8209-8213 c) Y. Li, Z. Wang, Org. Lett. 2009, 11, 1385-1387.

[8] W. Jiang, Y. Li, Z. Wang, Chem. Soc. Rev. 2013, 42, 6113-6127.

[9] M. Gingras, J.-M. Raimundo, Y. M. Chabre, Angew. Chem., Int. Ed. 2006, 45, 1686-1712; Angew. Chem. 2006, 118, 1718-1745.

[10] M. Bancu, A. K. Rai, P. Cheng, R. D. Gilardi, L. T. Scott, Synlett 2004, 173-176.

[11] J. H. R. Tucker, M. Gingras, H. Brand, J.-M. Lehn, J. Chem. Soc. Perkin Trans. 2 1997, 1303-1307.

[12] a) G. A. Downing, C. S. Frampton, D. D. MacNicol, P. R. Mallinson, Angew. Chem. Int. Ed. Engl. 1994, 33, 1587-1590; Angew. Chem. 1994, 106, 1653-1655; b) T. Baird, J. H. Gall, D. D. MacNicol, P. R. Mallinson, C. R. Michie, J. Chem. Soc., Chem. Commun. 1988, 1471-1473.

[13] Z. Wang, F. Doetz, V. Enkelmann, K. Müllen, Angew. Chem., Int. Ed. 2005, 44, 1247-1250, Angew. Chem. 2005, 117, 1273-1276.

[14] H. Qian, C. Liu, Z. Wang, D. Zhu, Chem Commun 2006, 4587-4589.

[15] B. D. Steinberg, E. A. Jackson, A. S. Filatov, A. Wakamiya, M. A. Petrukhina, L. T. Scott, J. Am. Chem. Soc. 2009, 131, 10537-10545.

[16] J. Heyd, G. E. Scuseria, M. Ernzerhof, J. Chem. Phys. 2003, 118 , 8207-8215.

[17] V. A. Rassolov, M. A. Ratner, J. A. Pople, P. C. Redfern, L. A. Curtiss, J. Comput. Chem. 2001, 22, 976-984.

[18] J. M. Hughes, Y. Hernandez, D. Aherne, L. Doessel, K. Müllen, B. Moreton, T. W. White, C. Partridge, G. Costantini, A. Shmeliov, M. Shannon, V. Nicolosi, J. N. Coleman, J. Am. Chem. Soc. 2012, 134 12168-12179.

[19] J. L. Segura, N. Martin, Angew. Chem., Int. Ed. 2001, 40, 1372-1409, Angew. Chem. 2001, 113, 1416-1453.

[20] P. T. Herwig, V. Enkelmann, O. Schmelz, K. Müllen, Chem. - Eur. J. 2000, 6, 1834-1839.

[21] Q. Zhang, P. Prins, S. C. Jones, S. Barlow, T. Kondo, Z. An, L. D. A. Siebbeles, S. R. Marder, Org. Lett. 2005, 7, 5019-5022. 


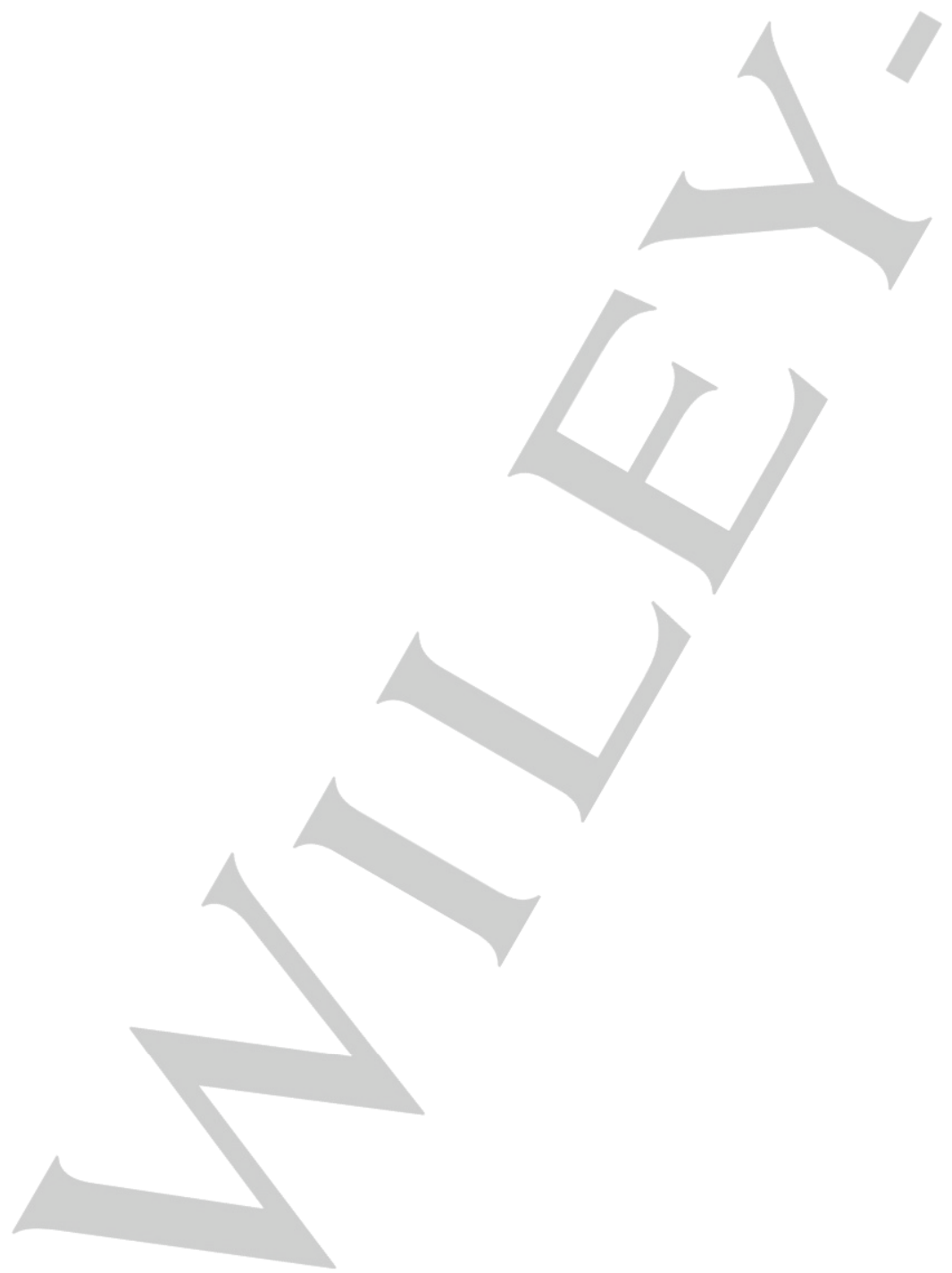




\section{COMMUNICATION}

Sulfur annulation: The

unprecedented sulfur annulated hexa-peri-hexabenzocoronenes were synthesized and unambiguously characterized by $X$ ray diffraction, revealing the distinct sulfur-annulated peripheral structure. Associated with DFT calculations, the strain minimization of the aromatic carbon skeleton should be responsible for the regioselective sulfur annulation at the bay positions.

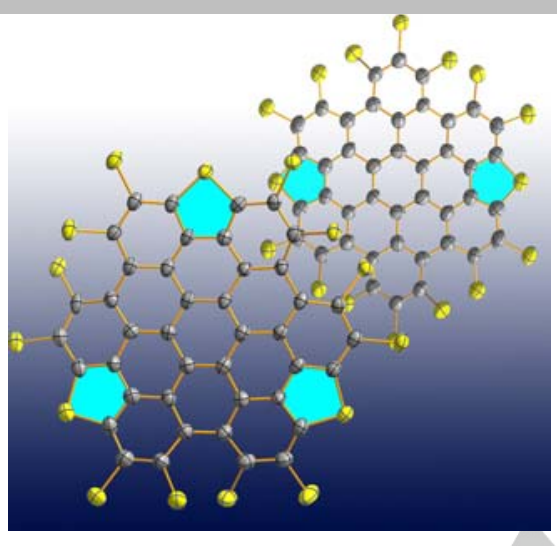

Yuan-Zhi Tan, Silvio Osella, Yi Liu, Bo Yang, David Beljonne, Xinliang Feng*, Klaus Müllen*

Page No. - Page No.

Sulfur Annulated Hexa-perihexabenzocoronene Decorated with Phenylthio Groups at the Periphery 